\title{
THE MULTIDISCIPLINARY APPROACH TO BREAST CANCER - THE ROLE OF SUPPORT SPECIALTIES BEFORE AND AFTER MASTECTOMY
}

\author{
Alexandra C. FILIP ${ }^{1 凶}$, Olivia A. MARCU ${ }^{2}$, Marius S. POP ${ }^{3}$, Daiana DEBUCEAN ${ }^{3}$ \\ ${ }^{1}$ Beauty Concept Clinic, Cluj-Napoca, Romania \\ ${ }^{2}$ Faculty of Medicine and Pharmacy, University of Oradea, Romania \\ ${ }^{3}$ Linea Medica Clinic, Oradea, Romania
}

Received 09 May 2020, Accepted 17 July 2020

hitps://doi.org/10.31688/ABMU.2020.55.3.09

\begin{abstract}
Introduction. Although many patients recover functionally, breast cancer and mastectomy have physical and psycho-emotional impact on the quality of life. To avoid errors and biases, reduce emotional distress and help postoperative recovery, physiotherapeutic and psychological assessments before and after mastectomy are needed.
\end{abstract}

The objective of the study was to demonstrate that physiotherapy and psychological counseling enhance the rehabilitation process, by assessing and treating the postural alterations and lymphedema and reducing the level of distress and anxiety.

Material and methods. We performed a prospective study on 20 patients with breast cancer, aged 32-65 years, divided into two equal groups. The study group undergone physiotherapeutic and psychological assessment and intervention, before mastectomy (for postural alterations, emotional status) and after mastectomy (for lymphedema, limited arm movements, anxiety, depression, hopelessness, acceptance). None of the patients in the control group presented to physiotherapeutic and psychological assessment before mastectomy. After surgery, all were assessed by a

\section{Résumé}

Lapproche multidisciplinaire du cancer du sein - le rôle des spécialités d'appui avant et après la mastectomie

Introduction. Bien que de nombreuses patientes se rétablissent fonctionnellement, le cancer du sein et la mastectomie ont un impact physique et psycho-émotionnel sur la qualité de la vie. Afin d'éviter les erreurs et les biais, réduire la détresse émotionnelle et aider à la récupération postopératoire, nous suggérons des évaluations physiothérapeutiques et psychologiques avant et après la mastectomie.

Objectif. De démontrer que la physiothérapie et le conseil psychologique améliorent le processus de rééducation, en évaluant et en traitant les altérations posturales et le lymphoedème et en réduisant le niveau de détresse et d'anxiété.

Méthodes. Il s'agit d'une étude prospective sur vingt patientes avec un cancer du sein âgées de 32 à 65 ans, réparties en 2 groupes égaux. Le groupe d'étude a subi une évaluation et une intervention physiothérapeutiques et psychologiques, avant (pour les altérations posturales, l'état émotionnel) et après la mastectomie 
physiotherapist, 2 patients completed the therapy; $70 \%$ went to psychological evaluation, but had poor attendance to counseling sessions.

Results. Six patients had postural and biomechanical changes and variable degrees of psychological distress, prior to mastectomy. The compliance to physiotherapeutic rehabilitation was greater in the study group than in the control group. The levels of distress were lower in the study group, due to pre-surgery counseling sessions.

Conclusions. The data confirmed the utility of preoperative assessments and counseling, the need for post mastectomy interventions and support the efficiency of the multidisciplinary approach of breast cancer cases.

Keywords: physiotherapy, psychological assessment, counseling, compensation, distress.

\section{Abbreviations:}

MMFBCP - Multidisciplinary Management File for Breast Cancer Patients

SG - study group

CG - control group (pour le lymphœdème et les mouvements limités du bras, l'anxiété, la dépression, le désespoir, l'acceptation). Aucune des patientes du groupe témoin ne s'est présentée à une évaluation physio-thérapeutique et psychologique avant la mastectomie. Après la chirurgie, toutes ont été évaluées par un physiothérapeute, 2 patientes ont terminé la thérapie. $70 \%$ ont participé à une évaluation psychologique après la mastectomie, mais avaient une faible fréquentation des séances de conseil.

Résultats. Six patientes ont eu des changement posturaux et biomécaniques et des degrés variables de détresse psychologique, avant la mastectomie. La conformité à la réadaptation physiothérapeutique était plus élevée dans le groupe d'étude que dans le groupe témoin. Les niveaux de détresse étaient plus faibles dans le groupe d'étude, en raison des séances de conseil préopératoires.

Conclusions. Les données confirment l'utilité des évaluations et des séances de conseil préopératoires, la nécessité d'interventions post-mastectomie et l'efficacité de l'approche multidisciplinaire dans la prise en charge des cas de cancer du sein.

Mots-clés: physiothérapie, évaluation psychologique, conseil, compensation, détresse.

or sexual dysfunctions ${ }^{6}$ are disorders they can heal with the help of physiotherapy and psychotherapy and counseling.

The physiotherapist performs the overall postural evaluation, the assessment of the biomechanics of the scapular belt and upper limbs and the compensation mechanisms. We recommend the complex assessment both before and after surgery, in order to have a benchmark and to correctly monitor the rehabilitation process.

The clinical psychologist performs a complex assessment of the patient, in order to detect and address any disorder, eventually recommending a psychiatric consultation. The psycho-emotional impact of a cancer diagnosis is influenced by the previous history of mental health and some personal traits of the patient. After finding out the medical diagnosis, the patient goes through a strong emotional distress, then tries to take control of her situation, by seeking information and appropriate help. Medical terminology, treatment options, side effects, prognosis are aspects that generate confusion and overwhelm the patient.

Pain is a common postoperative symptom, an unpleasant sensory and emotional experience, associated with a potential or actual tissue damage, described in terms of such injuries? ${ }^{7}$. However, its 
intensity is subjective, the response to pain is influenced by personality traits, emotional factors ${ }^{8}$ (anxiety, depression, low self-esteem, pessimism may amplify the pain to the point of catastrophizing), social factors (stress, social isolation, lack of perceived social support ) and individual variables (education, coping style to pain can influence the way it is perceived, attitude towards the experience of pain). Up to $68 \%$ of breast cancer patients report pain that persists for up to 3 months after mastectomy ${ }^{9}$ due either to the complications of the intervention and radiotherapy ${ }^{10}$, or to demographic variables or psychological distress ${ }^{11}$.

The objective of the study was to demonstrate that physiotherapy and psychological counseling enhance the rehabilitation process of patients with breast cancer, by assessing and treating the postural alterations and lymphedema and reducing the level of distress and anxiety, thus increasing the quality of the patients' life.

\section{Material AND MEthods}

This prospective study took place between September 2017 - August 2019, in parallel with the implementation study of the Multidisciplinary Management File for Breast Cancer Patients (MMFBCP) ${ }^{12}$, in the County Emergency Clinical Hospital Cluj-Napoca, Romania. 20 patients previously diagnosed with breast cancer (6 with fibrosarcoma, 5 with adenocarcinoma and 9 with infiltrative ductal carcinoma), aged 32-65 years, were divided into 2 groups: the study group (SG) - 10 patients in which the MMFBCP was used, and the control group (CG) - 10 patients in which the classic approach was applied, with interdisciplinary collaboration, but without using the MMFBCP.

The MMFBCP is an extensive tracking sheet developed by us and including the investigations, observations and recommendations of all the specialists involved in the breast cancer case management - oncologist, oncological surgeon, plastic and reconstructive surgeon, radiotherapist, medical imaging specialists, physical medicine and rehabilitation specialist, physiotherapist, psychologist or any other specialists needed. The patients who expressed their consent to the use of the File had to undergone all the recommended steps of it.

The inclusion criteria were:

- breast cancer suitable for further reconstruction;

- stage up to $\mathrm{T}_{2} \mathrm{~N}_{0} \mathrm{M}_{0}$;

- consent to participate in the study, signing an informed consent.

The exclusion criteria were mammary pathologies unable to be reconstructed and those surgically outdated.
A plastic and reconstruction surgeon, a physiotherapist and a clinical psychologist were involved in this study, as well as an oncological surgeon who collaborated whenever necessary, without being directly involved.

The physiotherapist performed the overall postural evaluation ${ }^{13}$, according to a methodology previously established in agreement with the other medical specialties involved. The data obtained in the anamnesis were corroborated with those already recorded in the MMFBCP. The static inspection was performed in orthostatism, in three planes, with anatomical landmarks appreciated in space, related to the gravitational line. In the anterior frontal plane, we assessed:

- the position of the head. The comparison between pre- and post-mastectomy assessment indicates whether a change found is prior surgery or it is acquired recently, through a compensatory mechanism. Otherwise we can erroneously consider that it is an asymmetry acquired postoperatively.

- the clavicular lines - in scapular or hemithorax pathologies, the clavicles descend below the horizontal line, on one side. The asymmetry is a very good landmark for framing and monitoring.

- the biacromial line is evaluated separately. The compensatory mechanisms for a possible loss of control over the entire affected hemibody are assessed. There can be a significant decrease, even collapse, a loss of stability of the scapular belt. It can be a lowered shoulder or it can be the whole torso, which can be misleading.

- waist triangles. The extensive chest surgery may lead very frequently to idiopathic scoliosis or a scoliotic change in the frontal plane.

- the bitrochanteric, knee and bimalleolar lines are compared to an ideal horizontal plane.

In the posterior frontal plane, we evaluated:

- the position of the head;

- the biacromial line - the scapular control and the scapulothoracic stability are analyzed. The scapulothoracic and the scapulohumeral dysfunction is a common complication after mastectomy, since the patient cannot use properly her arm and the whole area is destabilized. A distance between the spinal line and the internal scapular edge of more than $1.5 \mathrm{~cm}$ is a positive sign for scapular instability, according to the Lateral Scapular Slide Test ${ }^{14}$, an indicator that the arm "falls" forward, collapses. If an instability is found, the subacromial pain and the impossibility of using the arm over $90^{\circ}$ are due to this instability, which is due to the oedema. As long as there is a scapular instability, there is a regional problem reflected by compensatory attitudes throughout the posture, which can lead not only 
to static changes, but also to significant dynamic dysfunctions ${ }^{15}$;

- the waist triangles from a posterior perspective;

- the bitrochanteric line, respectively the line of the posterior-inferior iliac spines, the symmetry of the gluteal folds, the popliteal fossa and the bimalleolar line.

In the sagittal plane, the position of the head and all segments (scapular, lumbar, pelvis, lower limb) are reported with an ideal imaginary line that passes through the tragus - acromion - the 3rd lumbar vertebra - the middle of the knee - the scaphoid of the foot. If the patient is wearing glasses, she will wear glasses so as not to change her body position. If a plummet is used in the assessment, it will be approached to the patient's head up to the first point where it will touch the body (not necessarily the head). The optimal distance between that plummet and the farthest area of the cervical curvature is $6-8 \mathrm{~cm}$; if bigger, we may think of a head protrusion induced by an analgesic position. The farthest point from the lumbar curvature is ideally at $4-6 \mathrm{~cm}$. These landmarks are strictly indicative, they do not necessarily prove pathology, rather they help to clarify the moment change occurs (pre- or postoperative) and the eventual displacement found be put in a context. We suggest to take as many photos as possible during the assessment sessions, to monitor the postural changes. Thus, three important angles can be measured: the head, the cranio-cervical and the scapular angles. They provide valuable information about the state of the compensatory mechanisms, about evolution, about the musculoskeletal pains - they are all aggravated by these sudden changes, which inevitably involve the rest of the body: walking, stability, projection of the center of the body mass on the ground.

When assessing the transverse plane, two important lines are inspected: the biacromial / scapular and the bitrochanteric lines. We compare them with each other rather than with an ideal line. If these two lines deviate much from the physiological dominance (eye, arm, leg), their orientation is appreciated. If both lines are oriented much to the same side, it may be a pre-existing eye problem. If the scapular line is in a significantly opposite direction to the bitrochanteric line, the lower limb will be carefully examined, there is a possibility for the pelvis to enter a rotational compensation, helping the hemibody to perform satisfactorily daily activities or gestures; the spine begins to develop scoliosis not only frontally, but also transversely.

Dynamic postural inspection assesses:

- walking - the quality, speed and symmetry of the natural gait, possible analgesic and compensatory positions are observed, the patient's autonomy in walking and the compensatory movements.

- baropodometry - evaluates the distribution of body weight on the legs (front or rear, left-right symmetry),the loading on a certain leg (if there is an overload, a dominant, an anticipation of the step, an anticipation or a delay of the propulsion). A static change may be a constant dominant load due to compensations for oedema, pain or retractions).

- the autonomy in different contexts, involving complex body engrams, the use of all limbs, the change of the body from standing to sitting or bending or to maximum flexion of the knee (e.g. to simulate a daily activity). It is recommended to use the same dynamic tests in the preoperative and postoperative assessment.

- the degree of shoulder dysfunction: pain provocation tests (to locate suffering), facilitation tests (to determine biological resources) and functional tests (which have both diagnostic and monitoring purposes - e.g. the progress of the degree of flexion).

In the psychological evaluation we used:

- Depression, Anxiety and Stress Scales (DASS 21R $)^{16}$, a 21-item questionnaire that assesses negative emotional states associated with depression, anxiety and stress;

- The Body Image Scale ${ }^{17}$, a 10-item scale developed in collaboration with the European Organization for Cancer Research and Treatment (EORTC), based on the premise that the way the patient perceives her body is an important part of quality of life.

- The Freiburg Personality Inventory ${ }^{18}$, a multiphasic instrument of 12 scales that investigates life satisfaction, social orientation, achievement orientation, inhibition, excitability, aggression, solicitation, somatization, health care, sincerity, extraversion, emotionality.

\section{Results}

All patients in the SG attended the physiotherapeutic and psychological assessment sessions, before and after mastectomy. Six of them showed postural and biomechanical alterations and also muscle compensation mechanisms and attended physiotherapy sessions to correct to some degree the alterations. All of them participated in psychological counseling sessions before mastectomy, 4 sessions after mastectomy and 1 to 3 group meetings.

None of the 10 patients in the CG presented to physiotherapeutic and psychological assessment before mastectomy. After surgery, all of them were assessed by a physiotherapist, $80 \%$ started the 
The comparision of the spectrum of anxiety levels before and after mastectomy in the two groups
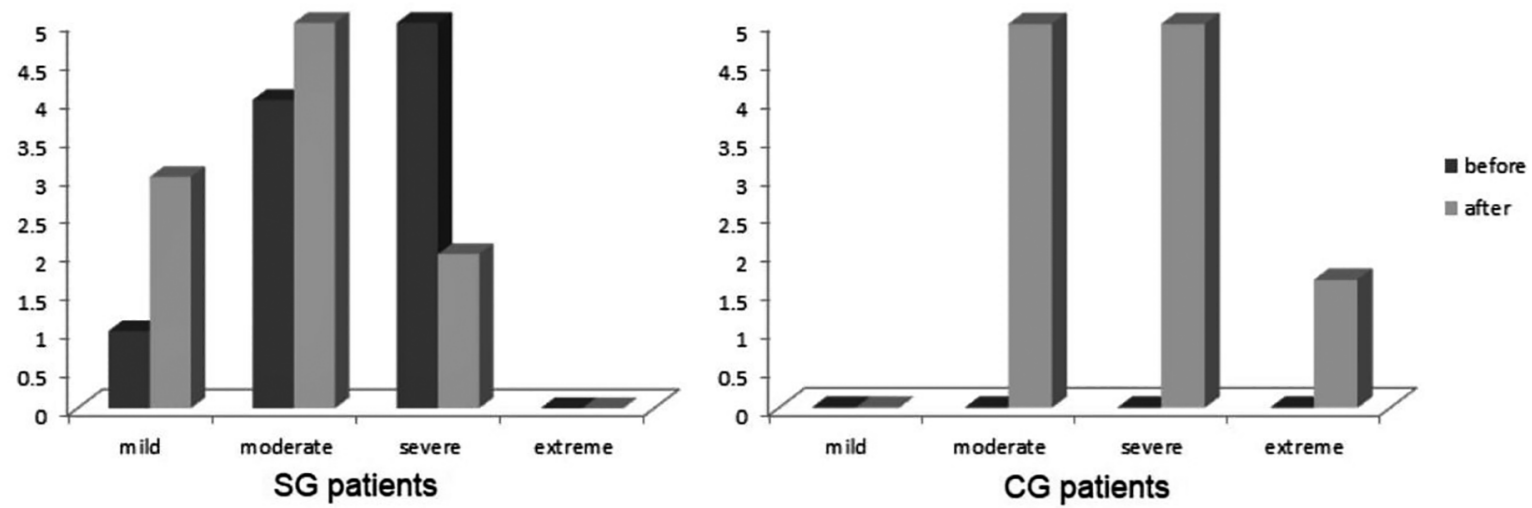

Figure 1. Anxiety levels before and after mastectomy for the SG patients versus CG patients

physiotherapy sessions and only 20\% completed the therapy. $70 \%$ of the patients went to psychological evaluation after the mastectomy, 3 attended 1 to 4 individual counseling and psychotherapy sessions and only one the group sessions.

Three patients of the SG and 5 of the CG had secondary lymphedema. All 3 patients in the SG, respectively 2 in the CG completed the proposed physical rehabilitation program.

In the SG, all patients showed symptoms of anxiety at the initial assessment, before mastectomy. Three patients were detected with symptoms of depression: 2 of mild intensity and one of moderate intensity (who benefited from psychiatric consultation). The stress level was high in all patients in the SG (scores between 10 and 16). All patients received counseling and therapy sessions prior to mastectomy. Subsequently, the postoperative evaluation showed a reduction in anxiety symptoms and stress levels and the same intensity depressive symptoms (Fig.1).

None of the patients of the CG presented for psychological evaluation before mastectomy. Postoperatively, all of the 7 patients assessed displayed symptoms of anxiety and depression (4 patients with mild depression and 3 moderate-intensity depression). Stress levels were increased in all of them, with scores 12 and 16.

The Body Image Scale administered after the mastectomy showed that young patients had several concerns about the changes throughout the treatment (breast loss, weight loss, hair loss, scars). Patients with a low level of education were more affected by possible bodily changes, developing defense mechanisms such as avoidance or denial, shame. There were no significant differences between batches.

Since all the patients declared themselves sincere, we noticed that postoperatively, the Freiburg Personality Inventory revealed that the only notable differences between the patients in the two groups were in terms of asthenic behavior, fear of illness and in emotionality (emotionally unstable, sensitive, with many worries). The tendency of excessive self-monitoring was greater in the CG patients (Fig. 2).

\section{Discussion}

In all these examinations, the patient was asked about the comfort she felt at that moment and context; if discomfort occurred, the evaluation was eventually discontinued. We did the evaluation in $2-3$ sessions; keeping the patient in the assessment situation for a long time generates fatigue, which can accentuate some lines or asymmetries or can distort the answers in the psychological assessment. We also noticed that some patients refused to make certain movements because they performed them asymmetrically and were ashamed to expose their asymmetry, the swollen arm, the scar or the inability to perform that movement. These are the so-called false tests. Therefore, the evaluator may be misled, as the tests may be significantly altered by the patient's emotional status. This can be overcome at the 2 nd or 3rd evaluation session, when the patient becomes familiar with the context and the evaluator.

All patients had shoulder dysfunctions of different degrees (painful, stiff, „frozen“ shoulder, with capsulitis), one of them a subluxated shoulder (due to loss of support from the deltoid). We explained to everyone that the lack of use caused the temporary loss of function. However, muscle structures have memory and the tone tends to recover, but the dynamics and fascia, the restricted tissues, cannot be overcome by a naturally recovered muscle structure, as the resistance is higher.

Lymphedema is a common consequence of mastectomy. A differential diagnosis was made with the 
The spectrum of the behaviours and attitudes measured

by the Freiburg Personality Inventory after mastectomy in the SG versus the CG

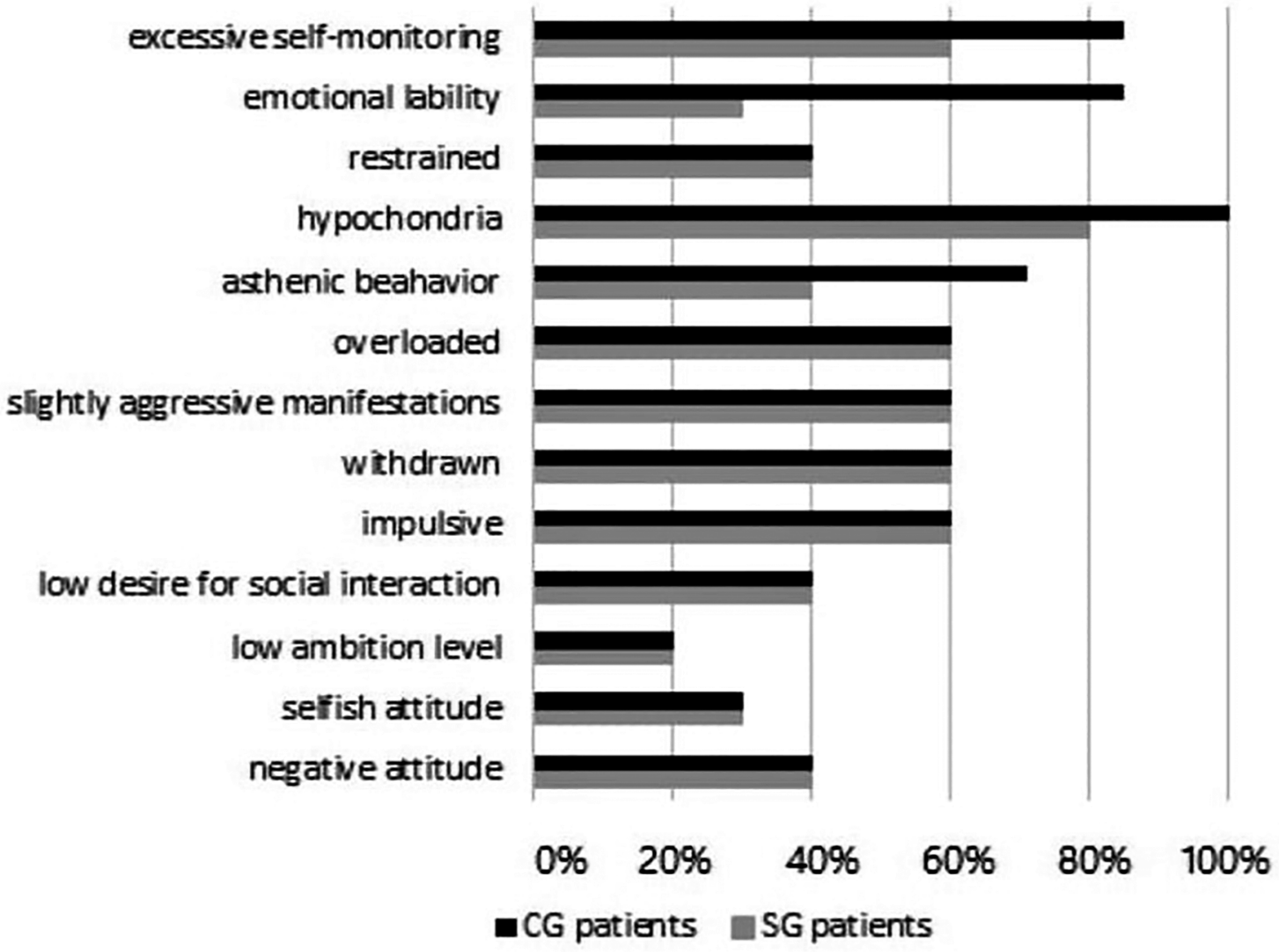

Figure 2. The distribution of the results in Freiburg Personality Inventory for SG versus CG patients

transient thoracic-outlet syndrome ${ }^{19}$, found in $2 \mathrm{pa}$ tients in the SG and one in the CG. It consisted of restrictions in the interscalene triangle, in the costal-clavicular space, in the subcoracoid space - the small pectoral muscle and in the cervical rib, due to the position of the head, which lead to vascular and nervous compressive phenomena, translated by paresthesia in the upper limb, distally. Beyond pain, there were motor problem ${ }^{20}$ which must be targeted in physical and functional rehabilitation.

Phlebitis is a common and very dangerous postoperative complication of mastectomy. The physiotherapist performed manual lymph drainage (has the advantage of perceiving the defense reaction of the tissue) and then applied a compressive bandage for up to 20 minutes for consolidation. Mechanical drainage, performed with devices, usually makes customization and feeling the response of the tissue (lymph dynamics and pain) impossible. Most of the patients had pain and did not report it ("I thought it was normal"), but the response to a painful therapy is generally more restriction.

Patients will most likely need to live a long time with the elastic glove with fingers to manage their minimal oedema and reduce the risk of phlebitis. At some point, the patient will stop drug therapy and weekly drainage, so the body has to be ready to function at its maximum potential. The physiotherapist, assisted by the psychologist, made sure that the patients understood the need to wear it, even if considered unsightly.

The physiotherapist made scar mobilization to restore elasticity, with the consent of the surgeon, as early as possible, gently, by stretching, because any incision, no matter how small, produces adhesions that may be an obstacle in the segmental rehabilitation.

The goal of the multidisciplinary team is to save these patients not only from death, but also from chronic, functional and psychological disability. Body segments should be functionalized and patients encouraged to resume normal activities prior to surgery. A patient's life expectancy depends on the resumption of all body functions, including the social function.

The therapy not only considered the muscular rehabilitation or the release of restrictions, but also the release of psycho-emotional obstacles that made the patient not use the arm (the fear of movement, 
the feeling of shame for appearance and functional incapacity). The psychotherapeutic approach was also very important, as starting with muscle recovery means pain and it discourages the patient.

Because the diagnosis of breast cancer, with all the therapeutic procedures involved, is perceived as a threat to life or to the integrity of the body, patients tended to over-self-monitor (a tendency to permanently scan and amplify "dangerous“ cues), consequently being extremely sensitive to the negative, dangerous, potentially painful aspects of the disease and treatment. Patients in the SG had a lower trend compared to the CG, which may be due to the preoperative training they received in counseling sessions and which increased the understanding of the pathology and immediate postoperative conditions and thus the compliance to therapy.

The psychological distress measured in the pre- and post-mastectomy psychological assessments showed differences in the SG: anxiety decreased significantly (the number of patients with low and moderate anxiety increased, decreasing that of patients with severe anxiety), as well as the perceived stress level (with 3 points). The patients better understood and accepted the oncological therapeutic approach, the confidence in the medical act increased (these were goals in preoperative psychological counseling). In comparison, the CG showed similar levels of anxiety pre- and post-mastectomy, but depressive symptoms were present in $100 \%$ of the assessed patients in mild (4) and moderate (3) forms, and the stress level was higher.

Following mastectomy, patients faced misalignment and asymmetry of the breasts, the absence of one or both breasts, unsightly scars of varying sizes, sensory changes in the breasts, the need for breast prosthesis, possible changes in limb mobility, lymphedema. Generally, chemotherapy comes with possible transient hair loss, body weight fluctuations, loss of appetite, skin symptoms, hot flashes, pain, neurological disorders ${ }^{21}$. These negative changes are beyond the patients' ability to control, and their impact on the mental level can be extremely high, especially in patients who have not been prepared to cope with them. Although, the patient may be well physically, but not emotionally, due to the perception of body image. Women generally tend to pay more attention and be more dissatisfied with their physical appearance ${ }^{22}$, which is exacerbated by events that affect them and so happened in the two groups, too. The loss of a breast is directly related to a woman's identity, sexuality and self-esteem. The psychological counseling sessions before and after mastectomy targeted exactly these issues.
No significant differences were found between the two groups in terms of distress caused by the perception of body image and also of the age at which the distress is greater: young patients were more affected, their concerns about the body were more intense. Self-esteem related to physical appearance has four components (attractiveness, physical strength, physical condition and physical competence), and breast cancer affects all of them ${ }^{23}$. There is an increased discrepancy between the standard and the reality in the mirror, and it acts in two ways: either suddenly, in the form of a shock (generating symptoms of posttraumatic stress disorder), or slowly, prolonged, a gradual increase in mental tension and distress. The first variant was observed in young patients and the second in older ones; the slow evolution could be more easily managed over time and slowed down.

A large part of women has difficulties re-adapting to life after a mastectomy. Self-acceptance (a protective factor against potential emotional disorders) and awareness are valuable internal resources we can rely on in developing coping strategies, accompanying the patient in the process of self-knowledge and acceptance of the changes her body undergoes ${ }^{24}$.

The physical and functional rehabilitation performed by the support specialties came to support the patient, as well as the other medical specialties involved. A patient with postural stability, balanced in all her body segments, but also emotionally balanced, had a better management of pain and surgery consequences, a good therapeutic compliance, thus increasing the chances of success of further treatment. We found that the success was due to the multidisciplinary collaboration and implicitly to the use of the Multidisciplinary Management Card for Breast Cancer Patients, which offered an overview and a unitary approach, visible in the results obtained.

\section{THE LIMITS OF THE STUDY}

The number of patients in the two groups is indeed low, but even so the results are relevant. There is still no standard protocol for this approach, therefore the decision belonged to the medical and support staff involved, with the voluntary participation and consent of patients.

\section{Conclusions}

Due to the complexity of this pathology, we recommend evaluation, therapy and rehabilitation in a multidisciplinary team, which maximizes the benefits of each stage. The literature documents with increasing accuracy the benefits of a patient-centered multidisciplinary rehabilitation, over a well-defined 
period of time and oriented towards the recovery of functions.

Although many patients recover functionally acceptable, the impact of breast cancer and mastectomy on the quality of everyday life is strong and lasting. They have to face physical, psycho-emotional and psychosocial obstacles, which may be overcome with the help of support specialties: physiotherapy and psychotherapy.

Global postural assessment, evaluation of the biomechanics of the scapular belts and upper limbs, as well as the compensation mechanisms are performed by the physiotherapist before the mastectomy (for benchmark) and after it (proper monitoring of the rehabilitation process, avoid misinterpretations). The psycho-emotional evaluation and subsequent counseling decreases the impact and emotional distress generated by diagnosis and therapy and supported a faster and complex recovery.

Patients who accepted this new approach were more compliant and had a better follow-up compared to those who were only partially and sporadically involved, choosing instead the classic approach.

\section{Authors' Contributions:}

Conceptualization, A.C.F., D.D., M.S.P; methodology, A.C.F. and O.A.M.; software, O.A.M.; validation, A.C.F. and D.D.; formal analysis, O.A.M.; investigation, A.C.F., D.D., M.S.P.; resources, A.C.F., D.D., M.S.P.; data curation, A.C.F., D.D., M.S.P.; writing - original draft preparation, A.C.F., D.D., M.S.P.; writing - review and editing, A.C.F. and D.D.; visualization, A.C.F., D.D., M.S.P., O.A.M.; supervision, A.C.F.; project administration, A.C.F., D.D., M.S.P. All the authors have read and agreed with the final version of the article.

\section{Compliance with Ethics Requirements:}

„The authors declare no conflict of interest regarding this article"

"The authors declare that all the procedures and experiments of this study respect the ethical standards in the Helsinki Declaration of 1975, as revised in 2008(5), as well as the national law. Informed consent was obtained from all the patients included in the study"

"No funding for this study"

\section{Acknowledgements: \\ None}

\section{References}

1. World Health Organization. The global burden of disease: 2004 update. Geneva: WHO. 2008:29-31.
2. Danaei G, Vander Hoorn S, Lopez AD, Murray CJ, Ezzati M. Causes of cancer in the world: comparative risk assessment of nine behavioural and environmental risk factors. Lancet. 2005;366:1784-1793.

3. Yip C, Smith RA, Anderson BO, et al. Guideline implementation for breast healthcare in low-and middle-income countries: early detection resource allocation. Cancer. 2009;113:2244-2256.

4. Wade DT. What is rehabilitation? An empirical investigation leading to an evidence-based description. Clin Rehabil. 2020; doi: 10.1177/0269215520905112.

5. Khan F, Amatya B, Ng L, Demetrios M, Zhang NY, Turner-Stokes L. Multidisciplinary rehabilitation for follow-up of women treated for breast cancer (Review). The Cochrane Library. 2012;12:3.

6. Khan F, Amatya B, Pallant JF, Rajapaksa I. Factors associated with long-term functional outcomes and psychological sequelae in women after breast cancer. The Breast. 2012; 21:314-320.

7. Merskey H, Bogduk N (eds). Classification of Chronic Pain. Seattle, IASP Press. 1994:209.

8. Lautenbacher S, Huber C, Schofer D, et al. Attentional and emotional mechanisms related to pain as predictors of chronic postoperative pain: a comparison with other psychological and physiological predictors. Pain. 2010;151:722-731.

9. Jung BF, Ahrendt GM, Oaklander AL, Dworkin RH. Neuropathic pain following breast cancer surgery: proposed classification and research update. Pain. 2003;104:1-13.

10. Gartner R, Jensen MB, Nielsen J, Ewertz M, Kroman $\mathrm{N}$, Kehlet $\mathrm{H}$. Prevalence of and factors associated with persistent pain following breast cancer surgery. JAMA. 2009;302:1985-1992.

11. Bruce J, Thornton AJ, Powell R, et al. Psychological, surgical, and sociodemographic predictors of pain outcomes after breast cancer surgery: a population-based cohort study. Pain. 2014; 155:232-243.

12. Baciu AC, Marcu OA, Pop MS, Debucean D. A multidisciplinary approach to breast cancer - introducing a management file for breast cancer patients. Arch Balk Med Union. 2020;55(1):80-86.

13. Mangone M, Bernetti A, Agostini F, et al. Changes in spine alignment and postural balance after breast cancer surgery: a rehabilitative point of view. Biores Open Access. 2019; $8(1): 121-128$

14. Odom CJ, Taylor AB, Hurd CE, Denegar CR. Measurement of scapular asymmetry and assessment of shoulder dysfunction using the lateral scapular slide test: a reliability and validity study. Physical Therapy. 2001;81(2):799-809.

15. Hadad CAS, Saad M, Janeiro Perez MC, Miranda F. Assessment of posture and joint movements of the upper limbs of patients after mastectomy and lymphadenectomy. Einstein (Sao Paulo). 2013;11(4):426-434.

16. Lovibond SH, Lovibond PF. Manual pentru scalele de depresie, anxietate și stres. Cluj Napoca, Editura ASCR, 2011:7.

17. Hopwood P, Fletcher I, Lee A, Al Ghazal S. A body image scale for use with cancer patients. European Journal of Cancer. 2001; 37: 189-197.

18. Fahrenber J, Selg H, Hampel R. Inventarul de personalitate Freiburg. București, D\&D Consultants Grup S.R.L., 2007.

19. Sanders RJ, Hammond SL, Rao NM. Diagnosis of thoracic outlet syndrome. Journal of Vascular Surgery. 2007; 46(3):601-604

20. Loh SY, Musa AN. Methods to improve rehabilitation of patients following breast cancer surgery: a review of systematic reviews. Breast Cancer: Targets and Therapy. 2015;7:81-98. 
21. Diaconu CC, Dediu GN, Iancu MA. Drug-induced arterial hypertension, a frequently ignored cause of secondary hypertension: a review. Acta Cardiologica 2018;73(6):511-517.

22. Stokes R, Frederick-Recascino C. Women's perceived body image: relations with personal happiness. J Women Aging. 2003;15:17-29
23. Fox KR, Corbin CB. The physical self-perception profile: development and preliminary validation. J Sport ExercPsychol. 1989;11:408-430.

24. Izydorczyk B, Kwapniewska A, Lizinczyk S, Sitnik-Warchulska K. Psychological resilience as a protective factor for the body image in post-mastectomy women with breast cancer. Int J Environ Res Public Health. 2018;15(6):1181. 